\title{
Maximal Pericardial Effusion Width
}

National Cancer Institute

\section{Source}

National Cancer Institute. Maximal Pericardial Effusion Width. NCI Thesaurus. Code C139048.

The measurement of the maximum separation between the parietal and visceral pericardia. 